\title{
OGLE-2016-BLG-0693LB: Probing the Brown Dwarf Desert with Microlensing
}

\author{
Y.-H. RYU ${ }^{1}$, A. UdALSKI ${ }^{2}$, J. C. YeE ${ }^{3}$
}

AND

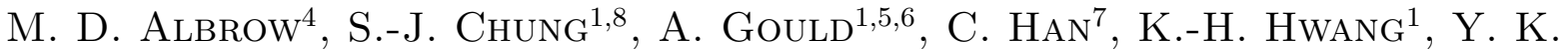

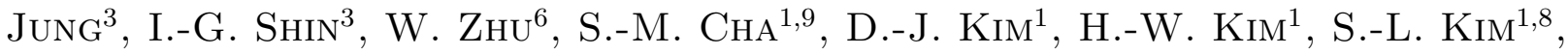

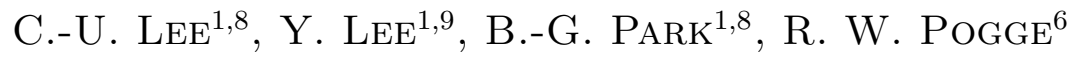

(The KMTNet Collaboration)

P. Pietrukowicz ${ }^{2}$, S. KozŁowski ${ }^{2}$, R. Poleski ${ }^{2,6}$, J. Skowron ${ }^{2}$, P. Mróz ${ }^{2}$, M. K. Szymański ${ }^{2}$, I. Soszyński ${ }^{2}$, M. PAWlak ${ }^{2}$, K. UlaczyK ${ }^{2}$

(The OGLE Collaboration)

${ }^{1}$ Korea Astronomy and Space Science Institute, Daejon 34055, Korea

${ }^{2}$ Warsaw University Observatory, Al. Ujazdowskie 4, 00-478 Warszawa, Poland

${ }^{3}$ Smithsonian Astrophysical Observatory, 60 Garden St., Cambridge, MA 02138, USA

${ }^{4}$ University of Canterbury, Department of Physics and Astronomy, Private Bag 4800, Christchurch 8020, New Zealand

${ }^{5}$ Max-Planck-Institute for Astronomy, Königstuhl 17, 69117 Heidelberg, Germany

${ }^{6}$ Department of Astronomy, Ohio State University, $140 \mathrm{~W} .18$ th Ave., Columbus, OH 43210, USA

${ }^{7}$ Department of Physics, Chungbuk National University, Cheongju 28644, Republic of Korea

${ }^{8}$ Astronomy and Space Science Major, Korea University of Science and Technology, Daejeon 34113, Korea

${ }^{9}$ School of Space Research, Kyung Hee University, Yongin, Kyeonggi 17104, Korea
\end{abstract}

\begin{abstract}
We present an analysis of microlensing event OGLE-2016-BLG-0693, based on the survey-only microlensing observations by the OGLE and KMTNet groups. In order to analyze the light curve, we consider the effects of parallax, orbital motion, and baseline slope, and also refine the result using a Galactic model prior. From the microlensing analysis, we find that the event is a binary composed of a
\end{abstract}


low-mass brown dwarf $\left(49_{-18}^{+20} M_{J}\right)$ companion and a K- or G-dwarf host, which lies at a distance $5.0 \pm 0.6 \mathrm{kpc}$ toward the Galactic bulge. The projected separation between the brown dwarf and its host star is less than $\sim 5 \mathrm{AU}$, and thus it is likely that the brown dwarf companion is located in the brown dwarf desert.

Subject headings: gravitational lensing: micro — binaries: general — brown dwarfs

\section{INTRODUCTION}

Brown dwarfs are generally known as failed stars that lack sufficient mass to ignite hydrogen burning. The upper mass limit of brown dwarfs so defined therefore varies from $0.07 M_{\odot}$ to $0.09 M_{\odot}$ according to the metallicity. On the other hand, the lower mass limit is defined by the onset of deuterium burning, i.e., about $13 M_{J}$ (Burrows et al. 2001). Due to their low luminosity, brown dwarfs can be hard to detect, particularly when distant from Earth. By contrast, the microlensing technique has an advantage to detect brown dwarfs at great distances, up to the Galactic bulge, because microlensing events can be detected regardless of the brightness of the lens system.

The discoveries of brown dwarfs can provide essential information on formation theory between giant planets and stars. Presently, 19 brown dwarfs have been detected by the microlensing method. Although the sample of microlensing brown dwarfs is still a small, it includes various types of brown dwarf systems such as isolated brown dwarfs (Poindexter et al. 2005; Gould et al. 2009; Zhu et al. 2016; Chung et al. 2017), brown dwarf companions to dwarf stars (Bachelet et al. 2012; Bozza et al. 2012; Shin et al. 2012a.,b; Park et al. 2013, 2015; Street et al. 2013; Jung et al. 2015; Ranc et al. 2015; Shvartzvald et al. 2016a), a brown dwarf binary (Choi et al. 2013), and a brown dwarf hosting a planet (Han et al. 2013; Shvartzvald et al. 2017).

Microlensing is particularly sensitive to detecting brown dwarfs around low-mass stars. Recently, Shvartzvald et al. (2016b) presented statistical results from 'second-generation' microlensing surveys by the Optical Gravitational Lensing Experiment (OGLE; Udalski et al. 2015), Microlensing Observations in Astrophysics (MOA; Bond et al. 2001), and Wise microlensing groups. They analyzed 224 microlensing events observed by all three teams during four observation-seasons, and suggested that the frequency of brown dwarf companions is relatively high in microlensing events. In addition, microlensing experiments are entering a new era, with surveys having wider fields of view and a higher cadence such as the Korea Microlensing Telescope Network (KMTNet; Kim et al. 2016). The KMTNet project was 
designed for the purpose of round-the-clock observing using three $1.6 \mathrm{~m}$ wide field optical telescopes located in Chile, South Africa, and Australia. Hence, all microlensing events are monitored at high cadence, making it possible densely monitor perturbations generated by brown dwarfs.

Due to the lack of discovery of brown dwarfs with respect to that of planets, there is a gap in the distribution between two populations of planets and stellar binaries that the so-called "brown dwarf desert". The brown dwarf desert exists over the mass-ratio range of $0.01<$ $q<0.1$ (Halbwachs et al. 2000; Chabrier 2003). Marcy \& Butler (2000) defined the brown dwarf desert as the paucity of brown dwarf companions to solar-type stars at separations of $<3$ AU. Grether \& Lineweaver (2006) quantified this desert as the intersection between two diverging power law mass functions, one for planets and one for stellar companions, which both decline toward brown dwarf masses. They therefore argued that the brown dwarf desert represents incontrovertible evidence for different formation mechanisms for stars and planets. However, the limits of the brown dwarf desert in separation and as a function of host mass are currently set by observational biases. Uncovering the true nature of this desert and its boundaries provides crucial information for understanding different formation mechanisms for stars and planets.

The microlensing method is optimal for probing the nature of the brown dwarf desert at intermediate separations $(1-10 \mathrm{AU})$ and exploring its outer edge, if one exists. Shvartzvald et al. (2016b) suggest that the brown dwarf desert might actually reach a minimum at SuperJupiter masses for their microlensing sample, which probes companions to $\mathrm{M}$ dwarfs at wider separations than RV studies. However, they state that more data are needed to confirm and characterize this minimum. The stellar companion mass function in this region is well understood (Duquennoy \& Mavor 1991; Raghavan et al. 2010), but the lower-mass bound of the desert has not been determined. Microlensing can complete our understanding of the brown dwarf desert by measuring the mass function of companions in this region into the planetary regime (Gould et al. 2010; Cassan et al. 2012; Suzuki et al. 2016). As with other techniques, microlensing is even more sensitive to brown dwarfs than to planets, and Gaudi (2002) suggested that $\sim 25 \%$ of brown dwarf companions from $1-10$ AU should be detectable in microlensing events if they exist. To date, planets have been the focus of microlensing, but a full analysis of all 2-body lens events will reveal the true extent of the brown dwarf desert and its implications for star and planet formation.

In this paper we report the discovery of a brown dwarf located in the brown dwarf desert from data taken by the OGLE and KMTNet survey experiments. The structure of this paper is as follows. In Section 2, we describe the discovery and survey observations, and present the light curve modeling in Section 3. In Section 4, we derive the physical properties 
of the lens system. In Section 5, we finish with a discussion of our findings.

\section{OBSERVATION}

OGLE-2016-BLG-0693 was announced as a new microlensing event by the OGLE Early Warning System (EWS) on 2016 Apr 15 UT 20:12 (Udalski et al. 1994; Udalski 2003; Udalski et al. 2015), and occurred on a star located toward the Galactic bulge with equatorial coordinates $(\alpha, \delta)_{\mathrm{J} 2000}=\left(17^{\mathrm{h}} 49^{\mathrm{m}} 55^{\mathrm{s}} .29,-23^{\circ} 2^{\prime} 23^{\prime \prime} .8\right)$ corresponding to Galactic coordinates $(l, b)=(5.548,2.220)$. OGLE observed using its $1.3 \mathrm{~m}$ Warsaw Telescope with a $1.4 \mathrm{deg}^{2}$ camera at the Las Campanas Observatory in Chile. This event was also observed by KMTNet and MiNDSTEp. In this paper, we used data based on the survey-only microlensing observations of the OGLE and KMTNet groups. KMTNet independently observed the event using three $1.6 \mathrm{~m}$ telescopes with $4 \mathrm{deg}^{2}$ camera in Chile (KMTC), South Africa (KMTS), and Australia (KMTA) (Kim et al. 2016).

In this study, we used the 491 OGLE data points taken over seven years, and the data of KMTC, KMTS, and KMTA (98, 124, and 59 points, respectively). Note that we removed several outlier data points for each data set. We re-scaled the photometry errors of the individual data sets in order to make $\chi^{2}$ per degree of freedom unity for the best-fit model. In the case of the KMTNet data, the event is located in a newly added field (BLG20) with the observation cadence of $\simeq 0.4 \mathrm{hr}^{-1}$ during 2016, and thus there are relatively few data points up to our adopted cut off of HJD'(HJD - 2450000) 7568. Despite the low observation cadence, the caustic entrance was detected by KMTS. We present the light curve of the event in Figure 1. The event has a caustic crossing feature of U-shape that is typical of binary lensing. Unfortunately, the caustic exit was not observed due to the weather conditions.

\section{BINARY LENS MODELING}

The slope in the baseline data is clearly apparent in Figure 1. This cannot be intrinsic to the source because it would imply a period of several decades, which would be completely inconsistent with the properties of such dim stars. On the other hand, such long term trends are a well known artifact induced by proper motions of neighboring bright stars. Therefore we include a linear trend in the blended flux to account for this effect.

In order to find all possible lensing models that can explain the light curve of the event,

we first conduct a grid search for $(s, q, \alpha)$ parameters, where $s$ is the projected separation between the companion and its host star, $q$ is the mass ratio of the two components of the lens 
system, and $\alpha$ is the angle of the source trajectory. In addition, the four lensing parameters $t_{0}, u_{0}, t_{\mathrm{E}}$, and $\rho$ are needed to fit a binary-lens light curve: $t_{0}$ and $u_{0}$ are respectively the time and separation of the source star's approach to a reference position (for which we adopt the center of mass), $t_{\mathrm{E}}$ is the Einstein ring crossing time corresponding to the total mass of the lens system, and $\rho$ is the source radius normalized to the angular Einstein radius $\left(\theta_{\mathrm{E}}\right)$. For a grid values of $(s, q, \alpha)$ parameters, we find the best-fitting lensing model by optimizing the other parameters using the Markov Chain Monte Carlo (MCMC) approach. For the case of $\alpha$, we seed it on a grid of six equally spaced starting values but then allow it to vary as a chain parameter to reduce calculation time. Since the major purpose of the grid search is to find crude solutions used for a starting point, we use a fixed value for the baseline slope of $0.03 \mathrm{yr}^{-1}$.

In Figure 2, we present the $\Delta \chi^{2}$ map for $(s, q)$ parameters, and identify two local solutions at close $(s<1)$ and wide $(s>1)$ separations, which is the well-known close/wide binary degeneracy (Dominik 1999). In the MCMC fitting process, we calculate the finitesource lensing magnification using the inverse ray-shooting method when the source passes the caustic (Kayser et al. 1986; Schneider \& Weiss 1988; Wambsganss 1997), while outside of the caustic, the magnification is calculated using the semi-analytic hexadecapole approximation (Pejcha \& Hevrovský 2009; Gould 2008). We also consider the limb-darkening effect by the source star. On the surface of the source star, the brightness profile is modeled by

$$
I_{\lambda}(\phi)=\frac{F_{0}}{\pi \theta_{*}^{2}}\left[1-\Gamma_{\lambda}\left(1-\frac{3}{2} \cos \phi\right)\right],
$$

where $F_{0}$ is the total flux of the source star, $\phi$ is the angle of the line of sight relative to the normal to the star's surface, and $\Gamma$ is the linear limb-darkening coefficient (Albrow et al. 1999). The limb-darkening coefficient $\left(\Gamma_{\lambda}\right)$ is related to the linear limb-darkening coefficient $u$ of Claret $(2000)$ as

$$
\Gamma_{\lambda}=\frac{2 u}{3-u} .
$$

We adopt $\Gamma_{\lambda}=0.54$ according to the source type (see Section 4 for more details).

For each local minimum of the grid search, we perform an optimization using the MCMC fitting process by allowing the eight parameters including the slope to vary. In the case of long timescale events, since the position of observer is changed due to Earth's orbital motion during the lensing phenomenon, the light curve is changed due to this parallax effect. Therefore we include the parallax effect in our model, and perform the fitting of the light curve by adding two parallax parameters $\pi_{\mathrm{E}, N}$ and $\pi_{\mathrm{E}, E}$ to the standard models with the slope (Gould 1992, 2004).

In Figure 3. we present the light curves and caustic structures for the best-fit parallax 
models at close $(s<1)$ and wide $(s>1)$ separations. The best fitting parameters and their $1 \sigma$ uncertainties are shown in Table 1, where the error of each individual parameter is the standard deviation determined from the distribution of MCMC chains. As shown in Table 1 , the lens system has a small mass ratio $(\sim 0.02)$, and has an unusually long timescale $t_{\mathrm{E}}$ and uncertainty compared to typical microlensing events.

Figure 4 shows that both parallax parameters are consistent with zero at the $2 \sigma$ level. It had been common practice in the past to suppress the parallax parameters in such cases on the grounds that the parallax was "not measured" to be different from zero. However, this viewpoint was not correct, as discussed in the Appendix of Han et al. (2016). The key point is that $\pi_{\mathrm{E}}$ is known a priori to be different from zero. so the fact that it is shown to be consistent with zero to within small errors is important information. In the present case, this parallax constraint will constrain the lens system not to be very close (and low mass). This will be combined with a flux constraint to provide reasonably strong constraints on all parameters. Hence this "non-measurement" actually plays an important role.

The question of whether to introduce orbital motion parameters is governed by the same fundamental issues of physics as for parallax parameters, but falls into a very different regime and therefore leads to a substantially different treatment. Just as we know a priori that all microlenses have finite (non zero) microlens parallax, we also know that all binaries have (non zero) orbital motion. Hence, we should in principle include such motion in the fit. We therefore begin by adding two linearized orbital motion parameters $d s / d t$ and $d \alpha / d t$, i.e., the instantaneous time derivatives of the separation and orientation of the binary axis. However, we find that these parameters are quite poorly constrained, with both the best fit and the majority of the chain being in the unphysical $\beta>1$ regime, with very modest improvement in $\chi^{2}$. Here $\beta$ is the ratio of the projected kinetic to projected potential energy (Dong et al. 2009) given by

$$
\beta=\left(\frac{\mathrm{KE}}{\mathrm{PE}}\right)_{\perp}=\frac{\kappa M_{\odot} \mathrm{yr}^{2}}{8 \pi^{2}} \frac{\pi_{\mathrm{E}} s^{3} \gamma^{2}}{\theta_{\mathrm{E}}\left(\pi_{\mathrm{E}}+\pi_{\mathrm{S}} / \theta_{\mathrm{E}}\right)^{3}}
$$

where $\pi_{\mathrm{S}}=\mathrm{AU} / D_{\mathrm{S}}$ is the parallax of the source star at the distance $D_{\mathrm{S}}, \gamma^{2}=(d s / d t / s)^{2}+$ $(d \alpha / d t)^{2}, \kappa=\left(4 G / c^{2} \mathrm{AU}\right) \simeq 8.14 \mathrm{mas} \mathrm{yr}^{-1}$, and $\pi_{\mathrm{E}}=\sqrt{\pi_{\mathrm{E}, N}^{2}+\pi_{\mathrm{E}, E}^{2}}$. We discuss the evaluation of $\theta_{\mathrm{E}}$ in Section 4.2 .

Since we know a priori that $\beta<1$, there is no information at all in the very mild preference of the models for large $\beta$. Under these circumstances, one might well consider suppressing orbital motion by imposing $d s / d t=d \alpha / d t=0$, i.e., simply not including these parameters in the fit.

However, in reality, all we are rigorously permitted to do by our prior knowledge is to 
impose the physical constraint $\beta<1$. In some circumstances, this would be effectively the same as ignoring orbital motion altogether (i.e., $d s / d t=d \alpha / d t=0$ ). However, as pointed out by Batista et al. (2011) and Han et al. (2016), orbital-motion parameters can be correlated with parallax parameters. Hence, they must be included with the $\beta<1$ constraint.

In fact we impose a constraint $\beta<0.8$ because larger values of $\beta$ require very unlikely physical situations observed at very special angles. We find that the parallax parameters are in this case essentially uncorrelated with the orbital motion parameters thus restricted, so that the basic result derived by suppressing orbital motion remains. However, the introduction of orbital motion parameters does slightly increase the uncertainty in the parallax. Hence, in our tables, we report results derived by including orbital motion parameters but restricted to $\beta<0.8$. We report the orbital motion parameters themselves in brackets because these values are not measurements but rather simply reflect the prior constraints.

\section{PHYSICAL QUANTITIES}

\subsection{Overview}

The total mass of lens system and its distance can be determined by

$$
M_{\mathrm{tot}}=\frac{\theta_{\mathrm{E}}}{\kappa \pi_{\mathrm{E}}} ; D_{\mathrm{L}}=\frac{\mathrm{AU}}{\pi_{\mathrm{E}} \theta_{\mathrm{E}}+\pi_{\mathrm{S}}} .
$$

If one applies Equation (4) to the chain results summarized in Table 1, the uncertainty in $M_{\text {tot }}$ would appear to be immense. First, since $\pi_{\mathrm{E}}$ is basically consistent with zero, the fractional error in $\pi_{\mathrm{E}}$ (and so $M_{\mathrm{tot}}$ ) should be almost infinite. In addition, for a microlensing event with a resolved caustic crossing, $\theta_{\mathrm{E}}$ is unusually poorly constrained. In the final analysis, this is due to the fact that the source brightness $I_{s}$ is very poorly constrained. As in the case of MOA-2011-BLG-293 (Yee et al. 2012), the large uncertainty in $I_{s}$ is due to the extreme faintness of the source. That is, for Einstein ring source positions $u \ll 1$, the solutions scale almost perfectly in $\left(f_{s}, \rho, q, u_{0}, 1 / t_{\mathrm{E}}\right)$. (Here $f_{s}$ is the OGLE source flux normalized at $I=18$.) Hence, almost all the information on breaking this degeneracy comes from portions of the light curve $u \sim 1$. However, because the source is extremely faint, the fractional errors in the magnified source flux are high. In addition, because this is a relatively low-cadence field for both OGLE and KMTNet, there are relatively few data points in this part of the light curve. If the source color is well-determined, then the source size $\theta_{*}=K \sqrt{f_{s}}$ where $K$ is a constant that we evaluate below. Then, $\theta_{\mathrm{E}}=\theta_{*} / \rho=K\left(f_{s} t_{\mathrm{E}}\right) /\left(t_{*} \sqrt{f_{s}}\right)$. Since (from Table 1$)$ the parameter combinations $\left(f_{s} t_{\mathrm{E}}\right)$ and $t_{*} \equiv \rho t_{\mathrm{E}}$ are relatively well constrained, this means that $\theta_{\mathrm{E}} \propto f_{s}^{-1 / 2}$. See also Yee et al. (2012). 
Nevertheless, there are two major pieces of auxiliary information that can constrain the physical nature of the system in conjunction with the microlens fit. First, there is a hard limit on the flux from the lens, which (together with assumption that the host is neither a neutron star nor a black hole), leads to a limit on the lens mass. Second, the lens and source are both drawn from Galactic populations whose statistical properties are known. In order to assess the individual impact of these two pieces of "external" information, we introduce them sequentially. However, first we evaluate $\theta_{*}$, or rather the constant $K$ that relates $\theta_{*}$ to the source flux $f_{s}$ of an individual solution.

\subsection{Evaluation of $\theta_{*}$}

Because (from Table 1) $I_{s}$ is very poorly determined, we begin by evaluating $\theta_{*}$ for an arbitrarily chosen fiducial value

$$
I_{s, \text { fid }}=22.50
$$

on the scale of the OGLE-IV observations. (As we discuss below, this fiducial value is much brighter than the best fit models in Table 1). We measure the centroid of the OGLE-IV clump to be at $I_{\text {clump }}=16.86$. We adopt $I_{0, \text { clump }}=14.35$ from Nataf et al. (2013) and thus derive

$$
I_{s, 0}=I_{s}+I_{0, \text { clump }}-I_{\text {clump }}=I_{s}-2.51,
$$

and hence $I_{0, s, \text { fid }}=19.99$.

Similarly, we find the dereddened source color in $(V-I)$ from its measured offset from the clump and the Bensby et al. (2013) evaluation $(V-I)_{0, \text { clump }}=1.06$. Unfortunately there are no magnified $V$ data from OGLE and only one significantly magnified $V$ point from KMT Chile (KMTC). To measure the source color we apply PyDIA (Albrow et al. 1999) to the KMTC data, which yields DIA light curves and photometry of neighboring stars on the same system 1 . We show in Figure 5, a calibrated version of the PyDIA color-magnitude diagram (CMD), which we obtain by aligning to OGLE-III (Szymański et al. 2011). Using PyDIA reductions of these data, we find an offset $(V-I)_{s}-(V-I)_{\text {clump }}=0.29 \pm 0.10$, and hence

$$
(V-I)_{s, 0}=(V-I)_{s}+(V-I)_{0, \mathrm{clump}}-(V-I)_{\mathrm{clump}}=1.35 \pm 0.10
$$

Finally, we apply the VIK color-color relations of Bessell \& Brett (1988) and the

\footnotetext{
${ }^{1}$ We note that while DoPhot (Schechter et al. 1993) has been demonstrated by Bensby et al. (2013) to work well for sources that become bright $(I<16)$ during the event, it may work less well for faint sources.
} 
color/surface-brightness relations of Kervella et al. (2004) to obtain

$$
\theta_{*}=(0.605 \pm 0.024) \mu \mathrm{as} \times 10^{-0.2\left(I_{0, s}-I_{0, s, \mathrm{fid}}\right)}=(0.605 \pm 0.024) \mu \mathrm{as} \times 10^{-0.2\left(I_{s}-22.50\right)}
$$

We note that a typical main-sequence star with $(V-I)_{s, 0}=1.35$, lying at the distance of the bar toward this direction $(7.4 \mathrm{kpc})$, would have a dereddened magnitude $I_{0} \sim 20.4$, a point to which we will return.

\subsection{Constraint on lens light}

The "baseline object" observed by OGLE has $I_{\text {base }}=20.53$, which $I_{\text {base }}-I_{\text {clump }}=3.98$ mag below the clump. This is the brightness of a bulge turnoff star. Since this baseline object must contain all the light from the source and lens (as well as other possible ambient stars), we conservatively conclude that the lens mass is $M<1.2 M_{\odot}$.

We then impose this constraint on the same chain that was summarized in Table 1. For each line in the chain, we take note of the values of $\left(I_{s}, \rho, \pi_{\mathrm{E}, N}, \pi_{\mathrm{E}, E}\right)$. We evaluate $\theta_{*}$ by inserting $I_{s}$ into Equation (8) and then evaluate $\theta_{\mathrm{E}}=\theta_{*} / \rho$ and $\pi_{\mathrm{E}}=\left(\pi_{\mathrm{E}, N}^{2}+\pi_{\mathrm{E}, E}^{2}\right)^{1 / 2}$. We insert $\theta_{\mathrm{E}}$ and $\pi_{\mathrm{E}}$ into Equation (44) and reject the link if the resulting value violates the mass constraint. Figure 6 presents the distribution of parallax parameters after the mass constraint, and Table 2 shows the resulting microlensing parameters.

Comparison of these results with Table 1 reveals several significant differences. Before commenting on these, however, it is important to note that the "conserved quantities" below the line actually change very little. As derived in Section 4.1, the fact that $\left(f_{s} t_{\mathrm{E}}\right)$ and

$t_{*}=\left(\rho t_{\mathrm{E}}\right)$ are nearly invariant, implies that $\theta_{\mathrm{E}}=K\left(f_{s} t_{\mathrm{E}}\right) /\left(t_{*} \sqrt{f_{s}}\right)$ basically scales $\propto f_{s}^{-1 / 2}$. Hence, it can be well approximated by

$$
M_{\mathrm{tot}} \propto f_{s}^{-1 / 2} \pi_{\mathrm{E}}^{-1} .
$$

From the first term, it follows that imposing a mass limit will tend to suppress solutions with small $f_{s}$ and therefore, from the approximate invariance of $\left(f_{s} t_{\mathrm{E}}\right),\left(q t_{\mathrm{E}}\right), t_{*}$, and $t_{\mathrm{eff}}$, also suppress solutions with large $t_{\mathrm{E}}$, small $q$, small $\rho$ and small $u_{0}$. The differences in these parameters between Tables 1 and 2 is uniformly a factor 2.2 for $s<1$ and 2.6 for $s>1$. We note that although the proper motion $\mu=\theta_{*} / t_{*}$ is not explicitly shown in these tables, it is directly affected, $\mu=\theta_{*} / t_{*} \propto f_{s}^{1 / 2}$, i.e., pushed upward.

The second term in Equation (9) directly suppresses solutions with small $\pi_{\mathrm{E}}$. Because (as is often the case) $\pi_{\mathrm{E}, E}$ is much better constrained by the light curve than $\pi_{\mathrm{E}, N}$, the main impact of the mass constraint is on $\pi_{\mathrm{E}, N}$ which then becomes approximately bimodal, 
especially for the $s<1$ solution. To better capture this behavior, we show in Table 2 the absolute value of $\pi_{\mathrm{E}, N}$, rather than the parameter itself.

\subsection{Galactic Model Prior}

The Galactic model prior is evaluated using the method of Batista et al. (2011), and the prior distribution of the Galactic model is given by

$$
P_{\mathrm{Gal}} \propto 2 R_{\mathrm{E}} v_{\mathrm{rel}} \nu(x, y, z) f(\boldsymbol{\mu}) g(M),
$$

where $R_{\mathrm{E}}$ is the physical Einstein ring radius, $v_{\text {rel }}=\mu D_{\mathrm{L}}$ is the lens velocity relative to the observer-source line of sight, $\boldsymbol{\mu}$ is the lens-source relative proper motion, $\nu(x, y, z)$ is the density distribution of the lenses. We adopt the Han \& Gould (2003) model which includes the bulge model of Dwek et al. (1995) and disk model of Zheng et al. (2001). Here $g(M)$ is the mass function, for which we adopt $g(M) \propto M^{-1}$ in common with Batista et al. (2011), and $f(\boldsymbol{\mu})$ is the probability function for the lens-source relative proper motion. Equation (10) can be expressed for microlensing parameters as

$$
P_{\text {Gal }} \propto \frac{4 D_{\mathrm{L}}^{4} \mu^{4}}{\mathrm{AU} \pi_{\mathrm{E}}} \nu(x, y, z) f(\boldsymbol{\mu})
$$

We apply the Galactic model priors to each of the chain links. We show microlensing parameters after imposing these priors in Table 3.

By comparing Table 3 with Table 2, we can understand the impact of these priors. The main impact comes from the Jacobian terms that are needed to transform Equation (10) to Equation (11). That is, as discussed by Batista et al. (2011) the microlensing chains have a uniform prior in $\boldsymbol{\pi}_{\mathrm{E}}$, for which phase space is dominated by short $D_{L}$, whereas for Euclidean geometry, phase space is dominated by large distances. This is origin of the terms " $D_{\mathrm{L}}^{4} \mu$ ". As discussed in Section 4.3, $\mu \propto f_{s}^{1 / 2}$. Hence, the $\mu^{4}$ term strongly favors higher $f_{s}$ and therefore also the corresponding changes in $\left(t_{\mathrm{E}}, q, \rho, u_{0}\right)$ implied by the invariant quantities at the bottom of Tables 1-3.

The $D_{\mathrm{L}}^{4}$ term works in the same direction, albeit more weakly. As shown in Section 4.1, $\theta_{\mathrm{E}} \propto f_{s}^{-1 / 2}$. Hence, high $f_{s}$ leads to lower $\theta_{\mathrm{E}}$, lower $\pi_{\mathrm{rel}} \equiv \theta_{\mathrm{E}} \pi_{\mathrm{E}}$, and so (via Equation (4)) higher $D_{\mathrm{L}}$. However, this effect is somewhat "watered down" by the appearance of $\pi_{\mathrm{S}}$ is the denominator. The $D_{\mathrm{L}}$ term similarly favors solutions with smaller $\pi_{\mathrm{E}}$, as of course does the direct appearance of $\pi_{\mathrm{E}}$ in the denominator of Equation (11).

All of these effects are seen in the comparison of Table 3 with Table 2. A more detailed analysis would show that the " $\nu(x, y, z)$ " and " $f(\boldsymbol{\mu})$ " terms act in the same direction, but 
more weakly. Comparing Table 3 to Table 1, one sees that the lens-light constraint and the Galactic priors combine to greatly reduce the range of microlensing parameters.

\subsection{Transformation from Microlensing to Physical Parameters}

In Figure 7, we present the histograms for the host $M_{1}$ and companion $M_{2}$ mass, the projected separation between them $a_{\perp}$, and the distance of the lens system $D_{\mathrm{L}}$. The histograms with black and red lines show the distributions of physical quantities from MCMC runs and the Galactic model prior including host-mass cut, respectively. In Table 4, we present the physical quantities with $1 \sigma$ error for two parallax+orbital motion models and applying the Galactic model prior (including host mass cut). In the case of the physical quantities obtained by applying the Galactic model prior, we mark them in brackets. The main effect of applying the priors is to shift the masses of the brown dwarf and host sharply higher and also to place the system at significantly larger distance. As discussed in Section 4.4, the primary reason for this is not the Galactic model parameters, but the Jacobian term, which in turn overwhelmingly reflects the ratio of phase space available to chain relative to the real physical phase space available to the lens.

In the present case, the mass cut (derived from the lens flux constraint) also plays a significant role. This is because the parallax measurement is consistent with zero, which means that the Jacobian itself tends to be unrestricted in its "preference" for high mass solutions. However, just as the microlens parallax cannot actually be zero, it also cannot be so small as to imply a huge lens mass, even though this is not ruled out by the MCMC chain itself.

Based on the results summarized in Table 4 and Figure 7, OGLE-2016-BLG-0693LB is well constrained to be a brown dwarf in either the close $(s<1)$ or wide $(s>1)$ solutions, which are fully degenerate (see first row of Table 1). Its host is almost certainly an FGK star, i.e., the traditional targets of RV surveys on which the brown dwarf desert was originally defined by Marcy \& Butler (2000), although there is a small probability that the host is an early M dwarf. The original definition of the "brown dwarf desert" ( $a<3 \mathrm{AU}$ ) was set by observational limits of RV surveys at that time. In the close solution, OGLE-2016-BLG0693LB is within the brown dwarf desert according to this restrictive definition. In the wide solution, it lies at or somewhat beyond its outer edge. This detection therefore illustrates the prospects for microlensing to probe the outer edges of the brown dwarf desert, where it is sensitive to both brown dwarfs and massive planets, and can thus determine whether the brown dwarfs remain a minimum in the companion mass function as they are at closer separations. 


\subsection{Two Consistency Checks}

The solutions derived in Tables 3 and 4 did not depend in any way on the source magnitude being "reasonable" given its color and only very weak prior information on the lens-source relative proper motion $\boldsymbol{\mu}$. That is, as we noted in Section 4.4, the Galactic prior does have a strong $\mu$ dependence, but this is overwhelmingly due to the Jacobian term $\mu^{4}$ and is only weakly responsive to the Galactic-model assumption $f(\boldsymbol{\mu})$. Therefore, we can ask whether the final values of these quantities are consistent with what is expected given our knowledge of the Galaxy, as external checks on the solution.

The best-fit source magnitudes in the $s<1$ and $s>1$ solutions are $I_{s}=22.3 \pm 0.4$ and $I_{s}=22.5 \pm 0.3$, respectively. Recall from Section 4.2 that the expected value for a typical star of the source's color and lying in Galactic bar would be $I_{s}=22.9$. Since variations in depth within the bar and source-star metallicity could cause this to vary by $\pm 0.3 \mathrm{mag}$, either of these models is consistent with expectations at the $1 \sigma$ level.

The model proper motions $(\mu=1.7 \pm 0.4$ and $\mu=1.4 \pm 0.2)$ at first sight appear more problematic given that the lens is in the disk and source is (at least assumed to be) in the bulge. This is because, if we assume ignore the peculiar motions of the observer, lens, and source, and if we assume a flat rotation curve and also assume zero bulk motion for the bulge, then the expected proper motion is just $\mu=v_{\text {rot }} / D_{\mathrm{S}}=6.3 \mathrm{mas} \mathrm{yr}^{-1}$, where we have adopted $v_{\text {rot }}=220 \mathrm{~km} \mathrm{~s}^{-1}$ and $D_{S}=7.4 \mathrm{kpc}$. Then it would appear to require some fine-tuning to "arrange" for the peculiar motions to almost perfectly cancel out this mean motion in order to yield $\mu \sim 1.5$ mas $\mathrm{yr}^{-1}$.

However, the Galactic longitude of this event is unusually high, $l=5.5^{\circ}$. The Galactic bar is certainly rotating. The exact angular speed is not precisely known, but we can plausibly adopt $100 \mathrm{~km} \mathrm{~s}^{-1} \mathrm{kpc}^{-1}$. Then the mean motion projected on the plane of the sky at the source position is $\sim 100 \mathrm{~km} \mathrm{~s}^{-1}$. For the geometry of this event (and now taking account of the Solar peculiar motion) the mean proper motion is $\overline{\boldsymbol{\mu}}=(3.0,0.0) \mathrm{mas} \mathrm{yr}^{-1}$ in Galactic $(l, b)$ coordinates. Note that in Equatorial coordinates, this direction is about $30^{\circ}$ East of North, which is very similar to the direction of $\boldsymbol{\pi}_{\mathrm{E}}$ (i.e., the same as $\boldsymbol{\mu}$ ). Given this mean motion, the observed proper motion is not at all unexpected.

Finally, we recall that $t_{*} \simeq 3.6 \mathrm{hr}$ is a near-invariant, i.e., it is a nearly model-independent property of the light curve. This long duration was actually essential to the publication of this paper. Recall that the event lies in a field with KMT cadence $\Gamma=0.4 \mathrm{hr}^{-1}$. It was only this long $t_{*}$ that permitted accurate measurement of the caustic crossing time. Even with the long $t_{*}$, there were only two data points over the caustic, which is the minimum for a reliable measurement. However, given that the great majority of microlensing sources are 
main-sequence stars (include the source star in the present case), such a long $t_{*}=\theta_{*} / \mu$ is only possible if the proper motion is small.

\section{CONCLUSION}

The event of OGLE-2016-BLG-0693 was discovered based on the survey-only microlensing observations of the OGLE and KMTNet groups. Although the event lies in a relatively low cadence KMTNet field $\left(0.4 \mathrm{hr}^{-1}\right)$, the caustic crossing part was observed by KMTS. This became possible because the source crossing time was exceptionally long (given that the source is a dwarf), $t_{*}=3.6 \mathrm{hr}$, which permitted two flux measurements over the caustic crossing. The long crossing time was in turn due to a proper motion, $\mu=1.55 \pm 0.3 \mathrm{mas} \mathrm{yr}^{-1}$, that is much lower than typical for most microlensing events, but not unexpected for events seen at high positive Galactic longitude $\left(5.5^{\circ}\right)$ on the near side of the Galactic bar.

In the analysis of the event, we considered the effect of parallax according to Earth's orbital motion during the lensing phenomenon because the event has an unusually long timescale (which is another consequence of its unusually low proper motion). In addition, we took the effects of baseline slope into account. We conducted the analysis of the light curve for two microlensing models with close and wide separation, respectively.

After applying a Galactic model prior and a lens-mass upper limit (due to limits on lens light), we found that the lens system is composed of a low-mass brown dwarf $\left(49_{-18}^{+20} M_{J}\right)$ orbiting a K or G dwarf. Finally, we note that the separation of the brown dwarf is less than $\sim 5 \mathrm{AU}$ demonstrating that microlensing is poised to probe the brown dwarf desert at intermediate separations.

We thank the anonymous referee for a very helpful report. This research has made use of the KMTNet system operated by the Korea Astronomy and Space Science Institute (KASI) and the data were obtained at three host sites of CTIO in Chile, SAAO in South Africa, and SSO in Australia. OGLE project has received funding from the National Science Centre, Poland, grant MAESTRO 2014/14/A/ST9/00121 to AU. AG, YKJ, and WZ were supported by US NSF grant AST-1516842. Work by IGS was supported by JPL grant 1500811. Work by C.H. was supported by the grant (2017R1A4A1015178) of National Research Foundation of Korea. YHR was supported by KASI (Korea Astronomy and Space Science Institute) grant 2017-1-830-03. 


\section{REFERENCES}

Albrow, M. D., Beaulieu, J.-P., Caldwell, J. A. R., et al. 1999, ApJ, 522, 1011

Bachelet, E., Fouqué, P., Han, C., et al. 2012, A\&A, 547, A55

Batista, V., Gould, A., Dieters, S., et al. 2011, A\&A, 529, A102

Bensby, T., Yee, J. C., Feltzing, S., et al. 2013, A\&A, 549, A147

Bessell, M. S., \& Brett, J. M. 1988, PASP, 100, 1134

Bond, I. A., Abe, F., Dodd, R. J., et al. 2001, MNRAS, 327, 868

Bozza, V., Dominik, M., Rattenbury, N. J., et al. 2012, MNRAS, 424, 902

Burrows, A., Hubbard, W. B., Lunine, J. I., \& Liebert, J. 2001, Reviews of Modern Physics, 73,719

Cassan, A., Kubas, D., Beaulieu, J.-P., et al. 2012, Nature, 481, 167

Chabrier, G. 2003, ApJ, 586, L133

Choi, J.-Y., Han, C., Udalski, A., et al. 2013, ApJ, 768, 129

Chung, S.-J., Zhu, W., Udalski, A., et al. 2017, ApJ, 838, 154

Claret, A. 2000, A\&A, 363, 1081

Dominik, M. 1999, A\&A, 349, 108

Dong, S., Gould, A., Udalski, A., et al. 2009, ApJ, 695, 970

Duquennoy, A., \& Mayor, M. 1991, A\&A, 248, 485

Dwek, E., et al. 1995, ApJ, 445, 716

Gaudi, B. S. 2002, arXiv:astro-ph/0206494

Gould, A. 2008, ApJ, 681, 1593-1598

Gould, A. 2004, ApJ, 606, 319

Gould, A. 1992, ApJ, 392, 442

Gould, A., Dong, S., Gaudi, B. S., et al. 2010, ApJ, 720, 1073 
Gould, A., Udalski, A., Monard, B., et al. 2009, ApJ, 698, L147

Grether, D., \& Lineweaver, C. H. 2006, ApJ, 640, 1051

Halbwachs, J. L., Arenou, F., Mayor, M., Udry, S., \& Queloz, D. 2000, A\&A, 355, 581

Han, C., \& Gould, A. 2003, ApJ, 592, 172

Han, C., Udalski, A., Gould, A., et al. 2016, ApJ, 828, 53

Han, C., Jung, Y. K., Udalski, A., et al. 2013, ApJ, 778, 38

Jung, Y. K., Udalski, A., Sumi, T., et al. 2015, ApJ, 798, 123

Kayser, R., Refsdal, S., \& Stabell, R. 1986, A\&A, 166, 36

Kervella, P., Thévenin, F., Di Folco, E., \& Ségransan, D. 2004, A\&A, 426, 297

Kim, S.-L., Lee, C.-U., Park, B.-G., et al. 2016, Journal of Korean Astronomical Society, 49, 37

Marcy, G. W., \& Butler, R. P. 2000, PASP, 112, 137

Nataf, D. M., Gould, A., Fouqué, P., et al. 2013, ApJ, 769, 88

Park, H., Udalski, A., Han, C., et al. 2015, ApJ, 805, 117

Park, H., Udalski, A., Han, C., et al. 2013, ApJ, 778, 134

Pejcha, O., \& Heyrovský, D. 2009, ApJ, 690, 1772

Poindexter, S., Afonso, C., Bennett, D. P., et al. 2005, ApJ, 633, 914

Ranc, C., Cassan, A., Albrow, M. D., et al. 2015, A\&A, 580, A125

Raghavan, D., McAlister, H. A., Henry, T. J., et al. 2010, ApJS, 190, 1

Schneider, P., \& Weiss, A. 1988, ApJ, 330, 1

Schechter, P. L., Mateo, M., \& Saha, A. 1993, PASP, 105, 1342

Shvartzvald, Y., Yee, J. C., Calchi Novati, S., et al. 2017, ApJ, in press, arXiv:1703.08548

Shvartzvald, Y., Li, Z., Udalski, A., et al. 2016a, ApJ, 831, 183

Shvartzvald, Y., Maoz, D., Udalski, A., et al. 2016b, MNRAS, 457, 4089 
Shin, I.-G., Han, C., Gould, A., et al. 2012a, ApJ, 760, 116

Shin, I.-G., Choi, J.-Y., Park, S.-Y., et al. 2012b, ApJ, 746, 127

Street, R. A., Choi, J.-Y., Tsapras, Y., et al. 2013, ApJ, 763, 67

Suzuki, D., Bennett, D. P., Sumi, T., et al. 2016, ApJ, 833, 145

Szymański, M. K., Udalski, A., Soszyński, I., et al. 2011, Acta Astron., 61, 83

Udalski, A. 2003, Acta Astron., 53, 291

Udalski, A., Szymański, M. K., \& Szymański, G. 2015, Acta Astron., 65, 1

Udalski, A., Szymanski, M., Kaluzny, J., et al. 1994, Acta Astron., 44, 227

Wambsganss, J. 1997, MNRAS, 284, 172

Yee, J. C., Shvartzvald, Y., Gal-Yam, A., et al. 2012, ApJ, 755, 102

Zheng, Z., Flynn, C., Gould, A., Bahcall, J. N., \& Salim, S. 2001, ApJ, 555, 393

Zhu, W., Calchi Novati, S., Gould, A., et al. 2016, ApJ, 825, 60 


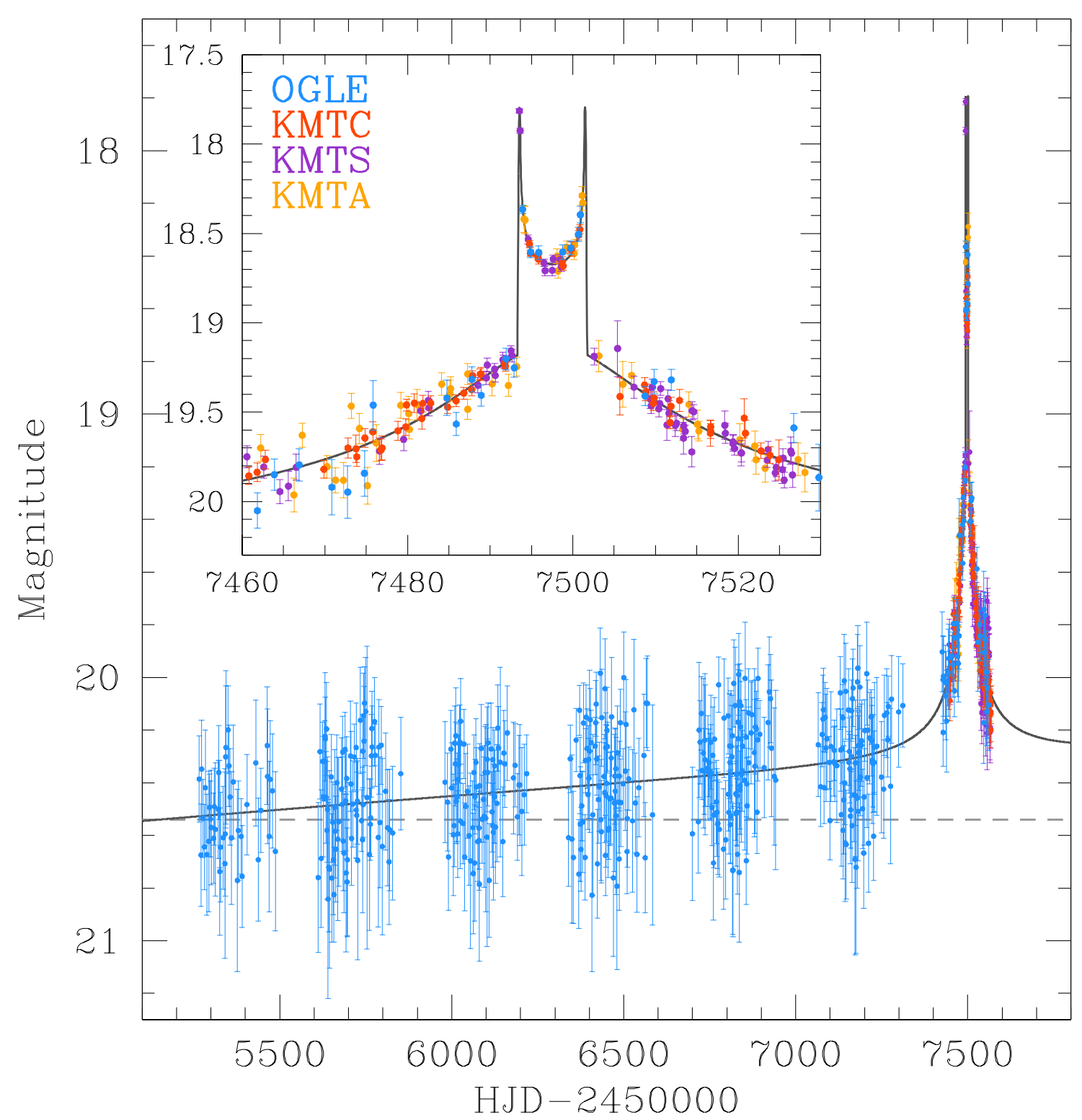

Fig. 1.- Full light curve data from OGLE (blue) and three KMTNet sets from KMTC (Chile, red), KMTS (South Africa, violet), and KMTA (Australia, yellow). Inset shows the data near the caustic crossing. Model light curve with black line includes the effect of baseline slope. 


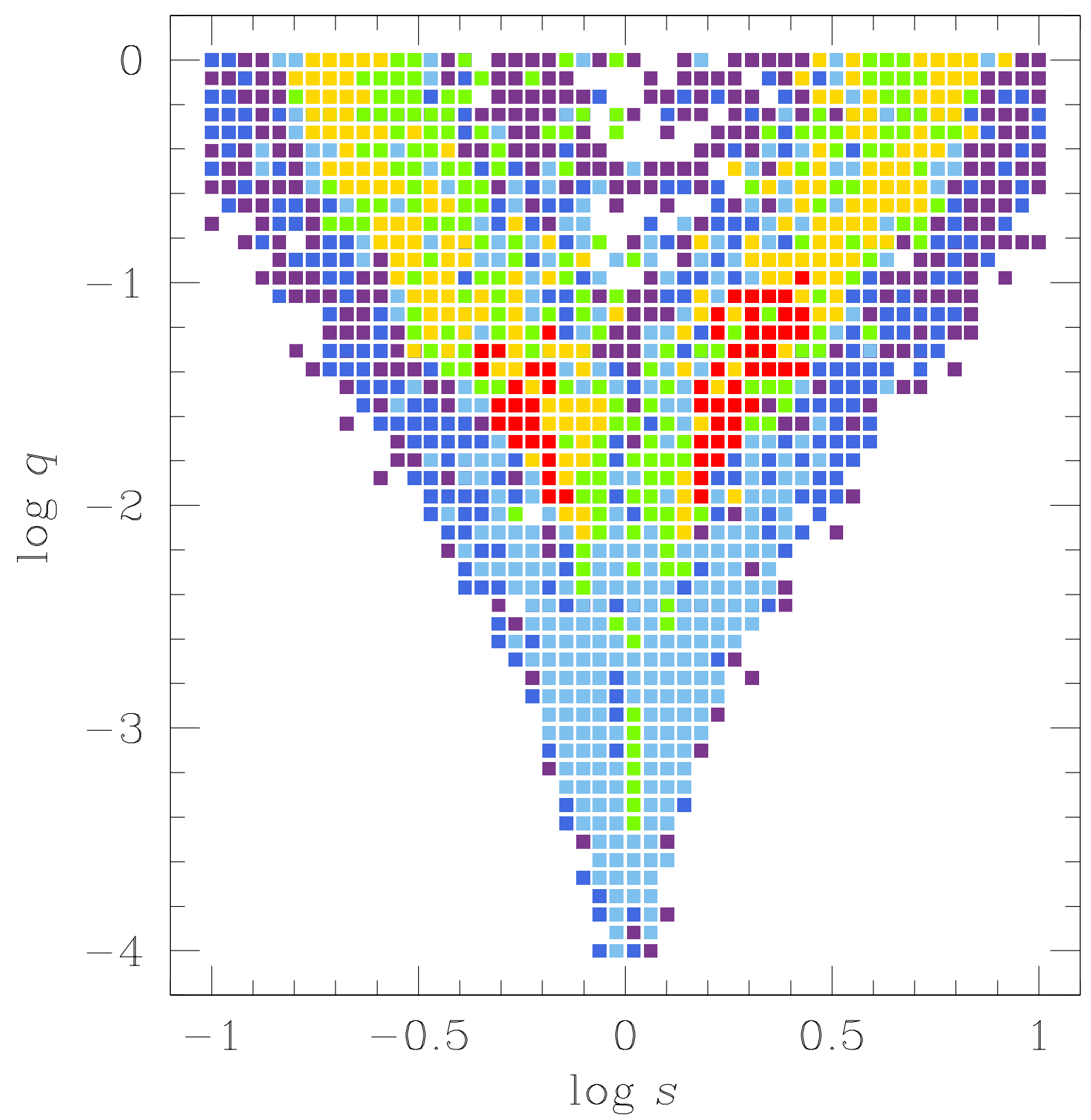

Fig. 2. - The $\Delta \chi^{2}$ map for $(s, q)$ parameters. The $\Delta \chi^{2}$ with $<100,<400,<900,<$ $1600,<2500$, and $<3600$ levels presented as red, yellow, green, light blue, blue, and purple, respectively. 

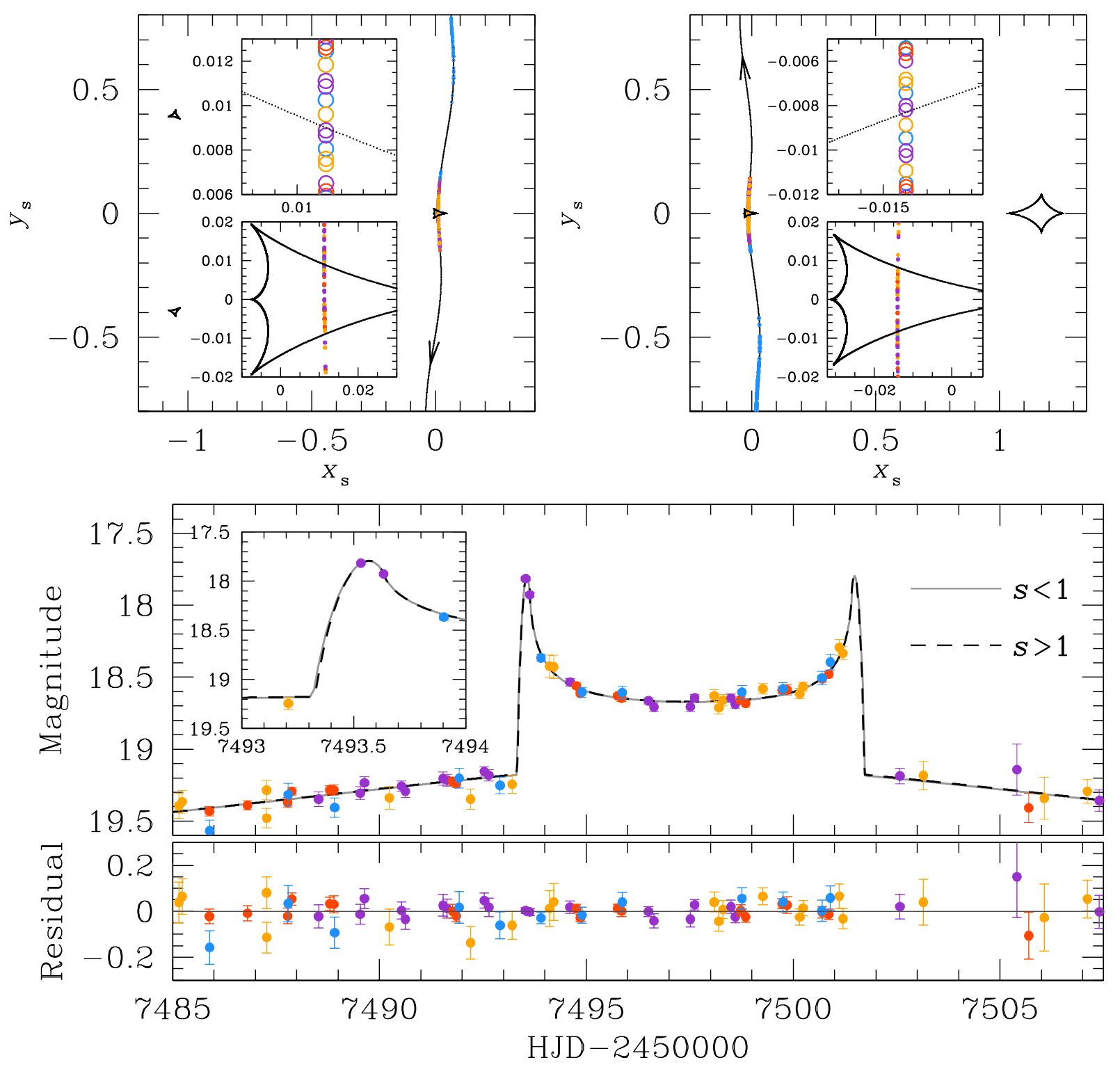

Fig. 3.- Caustic and light curve of the parallax models with the effect of slope. The upper two panels represent the caustic structure for the close- (left-hand panel) and wide-separation (right-hand panel) models. The black solid line with an arrow is the source trajectory, and the circles with various colors show the source positions corresponding to the time of observation of each observatory. The lower panel shows the light curves of the caustic crossing parts for close- and wide-separation models. 
Table 1. Best-fit solutions

\begin{tabular}{lccccc}
\hline \hline & \multicolumn{2}{c}{ Parallax models } & & \multicolumn{2}{c}{ Parallax+Orbital motion models } \\
\cline { 2 - 3 } \cline { 5 - 6 } Parameters & $s<1$ & $s>1$ & & $s<1$ & $s>1$ \\
\cline { 2 - 3 }$\chi^{2} /$ dof & $767.217 / 756$ & $767.304 / 756$ & & $766.769 / 754$ & $767.126 / 754$ \\
$t_{0}\left(\mathrm{HJD}^{\prime}\right)$ & $7497.484 \pm 0.087$ & $7497.559 \pm 0.158$ & & $7497.465 \pm 0.125$ & $7497.561 \pm 0.168$ \\
$u_{0}$ & $0.011 \pm 0.006$ & $0.014 \pm 0.010$ & & $0.010 \pm 0.010$ & $0.014 \pm 0.011$ \\
$t_{\mathrm{E}}($ days $)$ & $451.190 \pm 136.631$ & $488.108 \pm 287.923$ & & $507.369 \pm 127.955$ & $556.115 \pm 128.564$ \\
$s$ & $0.592 \pm 0.030$ & $1.760 \pm 0.044$ & & $0.582 \pm 0.039$ & $1.788 \pm 0.044$ \\
$q\left(10^{-2}\right)$ & $2.127 \pm 0.805$ & $2.077 \pm 1.532$ & & $1.931 \pm 1.261$ & $1.902 \pm 1.584$ \\
$\alpha(\mathrm{rad})$ & $1.562 \pm 0.019$ & $4.718 \pm 0.022$ & & $1.581 \pm 0.041$ & $4.719 \pm 0.022$ \\
$\rho\left(10^{-4}\right)$ & $3.296 \pm 1.462$ & $2.987 \pm 2.287$ & & $2.872 \pm 2.726$ & $2.689 \pm 2.426$ \\
$\pi_{\mathrm{E}, N}$ & $-0.017 \pm 0.125$ & $0.014 \pm 0.099$ & & $0.010 \pm 0.162$ & $0.003 \pm 0.141$ \\
$\pi_{\mathrm{E}, E}$ & $0.011 \pm 0.020$ & $0.012 \pm 0.019$ & & $0.011 \pm 0.027$ & $0.011 \pm 0.025$ \\
$\mathrm{Slope}\left(\mathrm{yr}{ }^{-1}\right)$ & $0.038 \pm 0.007$ & $0.038 \pm 0.008$ & & $0.037 \pm 0.009$ & $0.036 \pm 0.008$ \\
$d s / d t\left(\mathrm{yr} r^{-1}\right)$ & - & - & & {$[0.259 \pm 0.628]$} & {$[-0.107 \pm 0.471]$} \\
$d_{\alpha} / d t\left(\mathrm{yr}{ }^{-1}\right)$ & - & - & & $0.039 \pm 0.316]$ & {$[0.091 \pm 0.277]$} \\
$I_{s}$ & $23.649 \pm 0.378$ & $23.731 \pm 0.584$ & & $23.812 \pm 0.476$ & $23.882 \pm 0.433$ \\
$I_{b}$ & $20.587 \pm 0.039$ & $20.584 \pm 0.057$ & & $20.577 \pm 0.068$ & $20.573 \pm 0.057$ \\
\hline$t_{*}($ days $)$ & $0.148 \pm 0.021$ & $0.146 \pm 0.023$ & & $0.146 \pm 0.025$ & $0.149 \pm 0.021$ \\
$t_{\mathrm{eff}}($ days $)$ & $5.122 \pm 0.788$ & $6.812 \pm 1.608$ & & $4.817 \pm 0.809$ & $7.802 \pm 1.639$ \\
$q t_{\mathrm{E}}($ days $)$ & $9.597 \pm 1.187$ & $10.140 \pm 0.936$ & & $9.799 \pm 1.338$ & $10.579 \pm 0.935$ \\
$f_{\mathrm{s}} t_{\mathrm{E}}$ & $2.483 \pm 0.213$ & $2.491 \pm 0.189$ & & $2.402 \pm 0.218$ & $2.469 \pm 0.184$ \\
\hline
\end{tabular}

Note. - HJD' $=$ HJD -2450000 . $d s / d t$ and $d \alpha / d t$ cannot be regarded as "measured": They basically reflect the physically motivated condition $\beta<0.8$. 


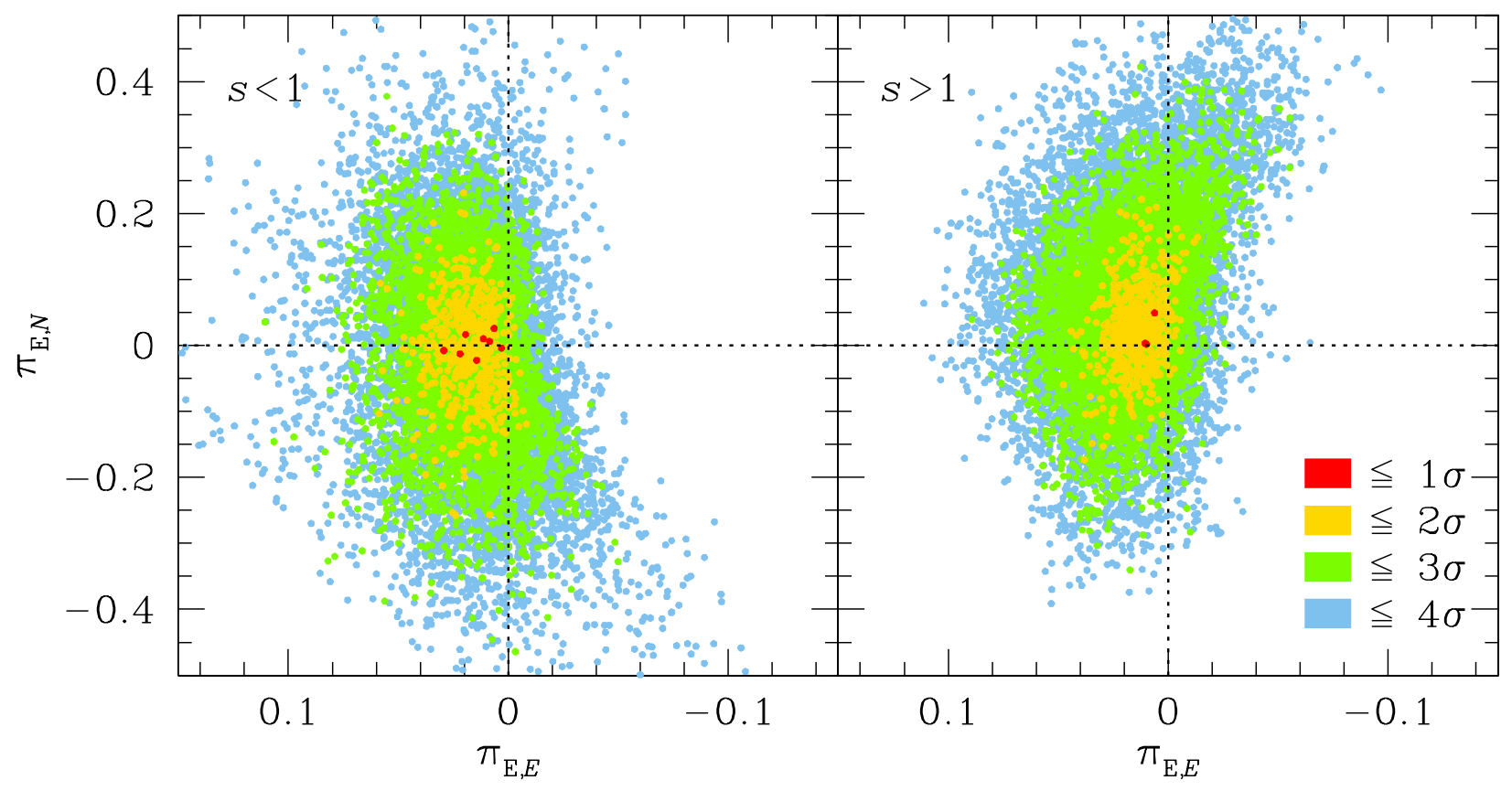

Fig. 4.- Contours for parallax parameters derived from MCMC fits. The colors of red, yellow, green, light blue correspond to $\Delta \chi^{2}<1,<4,<9$, and $<16$, respectively. 


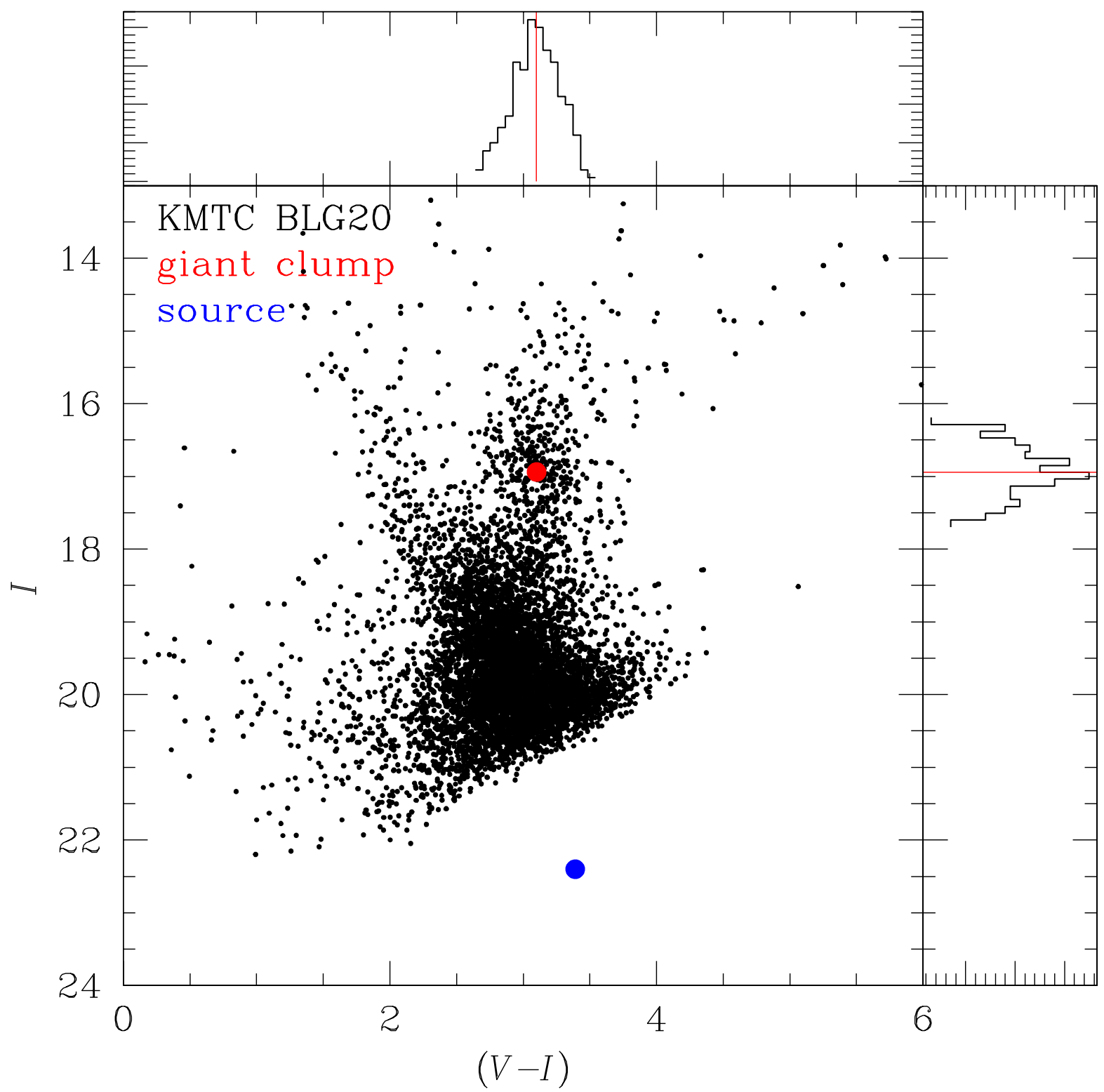

Fig. 5. - Color-magnitude diagram (CMD) of field stars around the source star in KMTNet CTIO BLG20 field. The CMD shows the microlensed source (blue) and centroid of the giant clump (red). The upper and right-side panels show the column density of stars around the giant clump. The algorithm centers on the 2-D peak and the histograms show 1-D distributions, which indicates good agreement. Note that the magnitude of the source flux is for the best fit, but the errors in this quantity are very large. See text for how these large error bars were handled. 


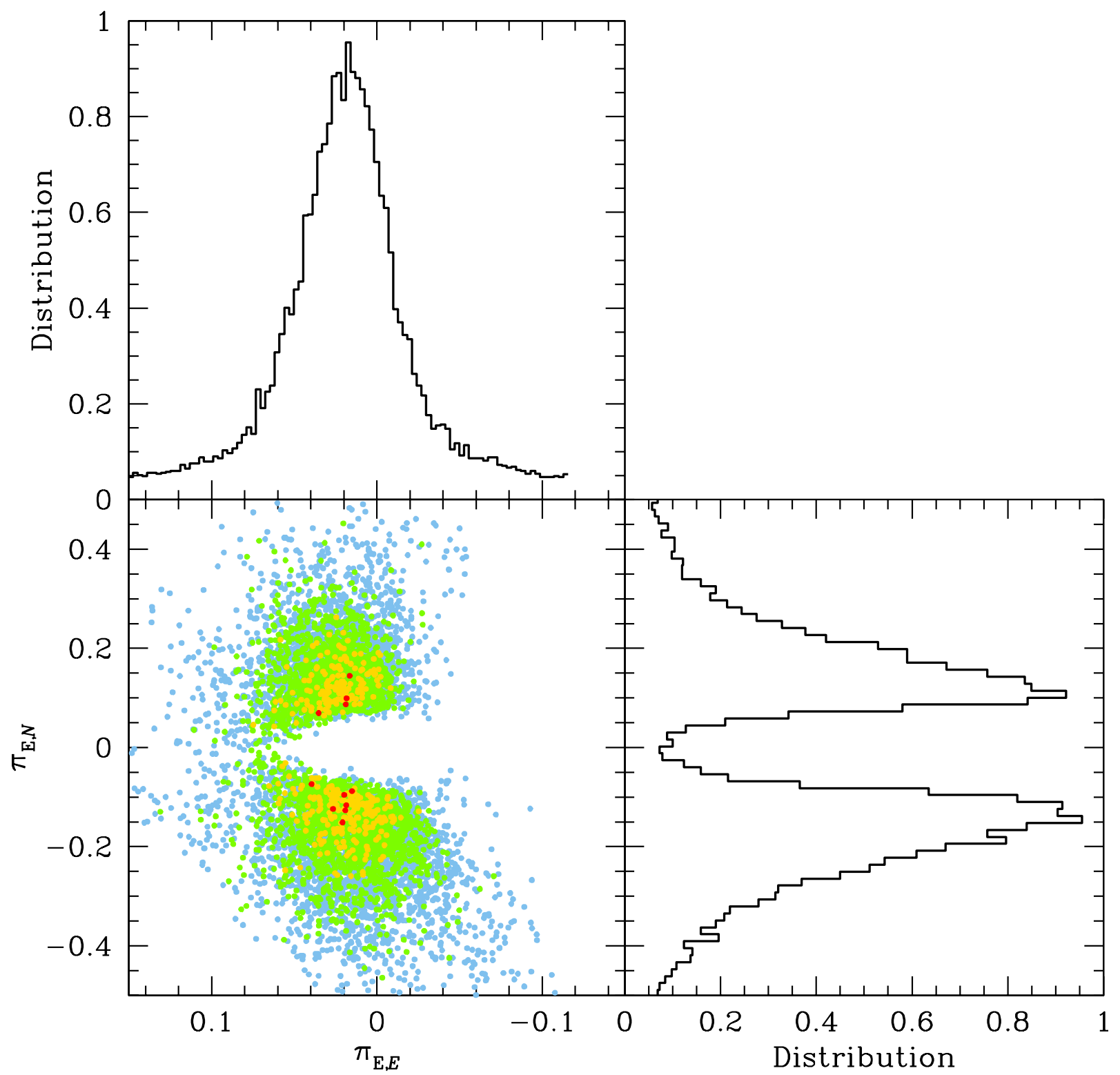

Fig. 6.- Contours and histograms for parallax parameters after the mass constraint. 
Table 2. LEnsing PARAMEters AFter the MASs CONSTRAint

\begin{tabular}{lcc}
\hline \hline & Parallax+Orbital motion models \\
\cline { 2 - 3 } Parameters & $s<1$ & $s>1$ \\
\hline$t_{0}\left(\mathrm{HJD}^{\prime}\right)$ & $7497.440 \pm 0.134$ & $7497.616 \pm 0.168$ \\
$u_{0}$ & $0.022 \pm 0.011$ & $0.029 \pm 0.011$ \\
$t_{\mathrm{E}}($ days $)$ & $244.540 \pm 103.180$ & $229.050 \pm 83.785$ \\
$s$ & $0.628 \pm 0.040$ & $1.754 \pm 0.047$ \\
$q\left(10^{-2}\right)$ & $3.705 \pm 1.321$ & $4.754 \pm 1.542$ \\
$\alpha(\mathrm{rad})$ & $1.598 \pm 0.044$ & $4.736 \pm 0.023$ \\
$\rho\left(10^{-4}\right)$ & $6.101 \pm 2.828$ & $6.892 \pm 2.357$ \\
$\left|\pi_{\mathrm{E}, N}\right|$ & $0.165 \pm 0.088$ & $0.159 \pm 0.091$ \\
$\pi_{\mathrm{E}, E}$ & $0.018 \pm 0.029$ & $0.016 \pm 0.029$ \\
Slope $\left(\mathrm{yr}^{-1}\right)$ & $0.047 \pm 0.008$ & $0.047 \pm 0.007$ \\
$d s / d t\left(\mathrm{yr}^{-1}\right)$ & {$[0.589 \pm 0.712]$} & {$[-0.672 \pm 0.498]$} \\
$d \alpha / d t\left(\mathrm{yr}{ }^{-1}\right)$ & {$[-0.522 \pm 0.307]$} & {$[-0.228 \pm 0.303]$} \\
$I_{s}$ & $22.949 \pm 0.469$ & $22.841 \pm 0.368$ \\
$I_{b}$ & $20.660 \pm 0.068$ & $20.675 \pm 0.054$ \\
\hline$t_{*}($ days $)$ & $0.151 \pm 0.024$ & $0.161 \pm 0.020$ \\
$t_{\mathrm{eff}}($ days $)$ & $5.533 \pm 0.989$ & $6.770 \pm 1.738$ \\
$q t_{\mathrm{E}}($ days $)$ & $8.807 \pm 1.326$ & $10.926 \pm 0.956$ \\
$f_{\mathrm{s}} t_{\mathrm{E}}$ & $2.616 \pm 0.272$ & $2.686 \pm 0.182$ \\
\hline & &
\end{tabular}


Table 3. Lensing Parameters weighted by the Galactic model Prior

\begin{tabular}{lcc}
\hline \hline & \multicolumn{2}{c}{ Parallax+Orbital motion models } \\
\cline { 2 - 3 } Parameters & $s<1$ & $s>1$ \\
\hline$t_{0}\left(\mathrm{HJD}^{\prime}\right)$ & $7497.451 \pm 0.123$ & $7497.555 \pm 0.156$ \\
$u_{0}$ & $0.039 \pm 0.013$ & $0.037 \pm 0.010$ \\
$t_{\mathrm{E}}($ days $)$ & $142.510 \pm 50.025$ & $169.250 \pm 42.235$ \\
$s$ & $0.677 \pm 0.039$ & $1.731 \pm 0.055$ \\
$q\left(10^{-2}\right)$ & $5.174 \pm 1.100$ & $6.229 \pm 1.525$ \\
$\alpha(\mathrm{rad})$ & $1.607 \pm 0.044$ & $4.736 \pm 0.019$ \\
$\rho\left(10^{-4}\right)$ & $9.838 \pm 3.124$ & $8.959 \pm 2.378$ \\
$\left|\pi_{\mathrm{E}, N}\right|$ & $0.087 \pm 0.048$ & $0.078 \pm 0.043$ \\
$\pi_{\mathrm{E}, E}$ & $0.029 \pm 0.034$ & $0.032 \pm 0.029$ \\
$\mathrm{Slope}\left(\mathrm{yr}{ }^{-1}\right)$ & $0.053 \pm 0.010$ & $0.050 \pm 0.008$ \\
$d s / d t\left(\mathrm{yr}{ }^{-1}\right)$ & {$[0.661 \pm 0.705]$} & {$[-0.912 \pm 0.346]$} \\
$d \alpha / d t\left(\mathrm{yr}{ }^{-1}\right)$ & {$[-0.627 \pm 0.258]$} & {$[-0.288 \pm 0.317]$} \\
$I_{\mathrm{S}}$ & $22.317 \pm 0.372$ & $22.547 \pm 0.289$ \\
$I_{\mathrm{b}}$ & $20.764 \pm 0.086$ & $20.722 \pm 0.059$ \\
\hline$t_{*}($ days $)$ & $0.145 \pm 0.024$ & $0.154 \pm 0.020$ \\
$t_{\mathrm{eff}}($ days $)$ & $5.632 \pm 0.518$ & $6.105 \pm 1.915$ \\
$q t_{\mathrm{E}}($ days $)$ & $7.576 \pm 1.109$ & $10.543 \pm 1.000$ \\
$f_{\mathrm{s}} t_{\mathrm{E}}$ & $2.661 \pm 0.144$ & $2.602 \pm 0.158$ \\
\hline
\end{tabular}


Table 4. Physical Properties of the Binary System

\begin{tabular}{lcc}
\hline \hline Quantity & $s<1$ & $s>1$ \\
\hline$M_{1}\left[M_{\odot}\right]$ & $0.59_{-0.28}^{+0.37}$ & $0.57_{-0.26}^{+0.37}$ \\
(prior) & $\left(0.86_{-0.22}^{+0.24}\right)$ & $\left(0.89_{-0.30}^{+0.22}\right)$ \\
$M_{2}\left[M_{J}\right]$ & $21.39_{-8.20}^{+12.84}$ & $25.41_{-10.47}^{+18.42}$ \\
(prior) & $\left(42.62_{-16.29}^{+18.22}\right)$ & $\left(54.75_{-1.25}^{+21.45}\right)$ \\
$D_{\mathrm{L}}[\mathrm{kpc}]$ & $3.64_{-0.97}^{+0.99}$ & $3.82_{-0.97}^{+1.07}$ \\
$($ prior $)$ & $\left(4.96_{-1.02}^{+0.64}\right)$ & $\left(5.19_{-0.87}^{+0.58}\right)$ \\
$a_{\perp}[\mathrm{AU}]$ & $1.85_{-0.48}^{+0.45}$ & $5.12_{-1.38}^{+1.25}$ \\
$($ prior $)$ & $\left(2.24_{-0.35}^{+0.32}\right)$ & $\left(5.80_{-0.80}^{+0.64}\right)$ \\
$\mu[\mathrm{mas} / \mathrm{yr}]$ & $1.21_{-0.28}^{+0.40}$ & $1.15_{-0.23}^{+0.24}$ \\
(prior) & $\left(1.62_{-0.36}^{+0.45}\right)$ & $\left(1.38_{-0.23}^{+0.28}\right)$ \\
\hline
\end{tabular}



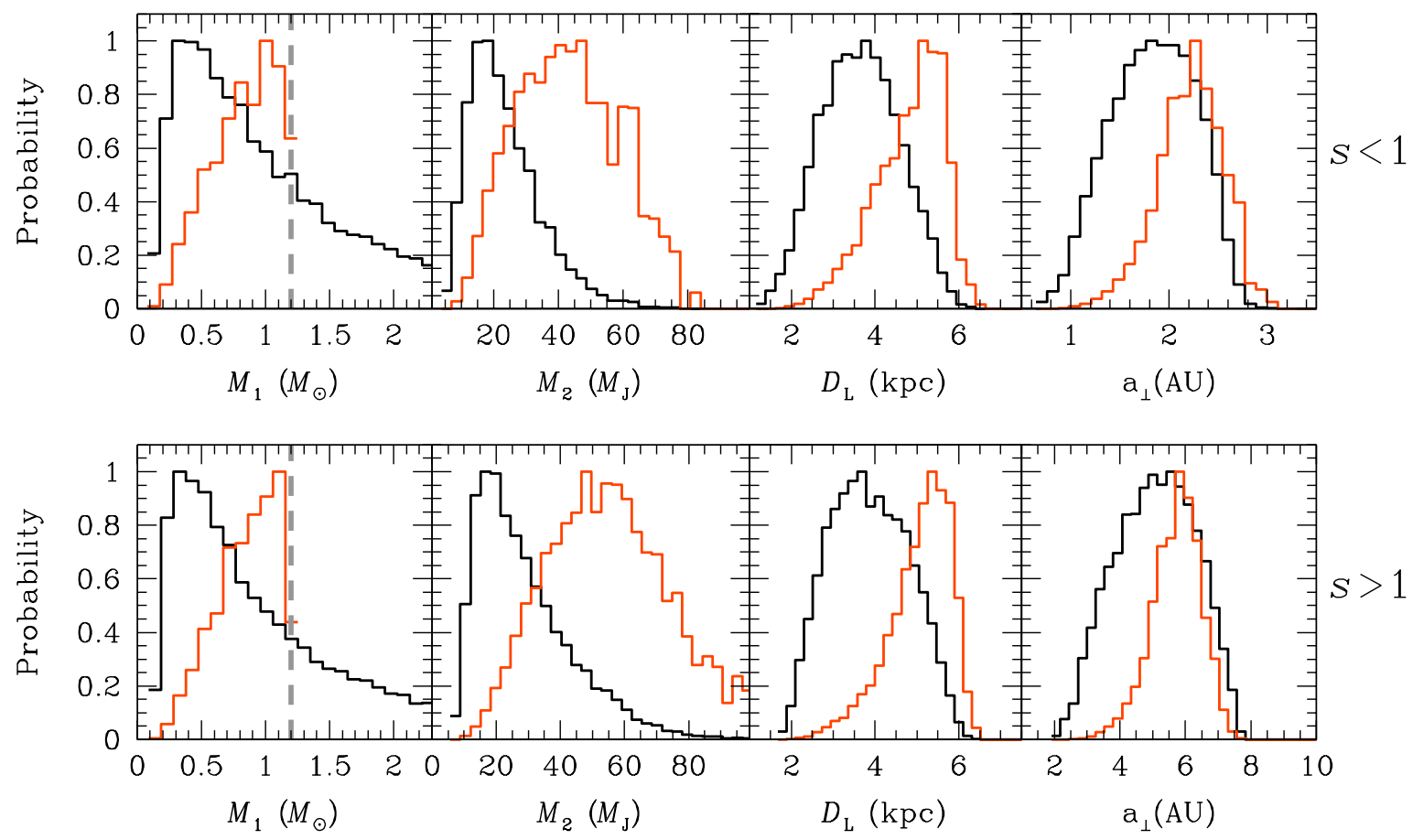

Fig. 7.- Histograms for physical quantities of the lens. The histograms with the black line represent the uncertainties of physical quantities from the MCMC runs, and the histograms with red line show that after applying the Galactic model prior (including the lens-mass cut $M<1.2 M_{\odot}$; gray dashed line). Large offsets between the black and red histograms is overwhelmingly due to the Jacobian term, which is the ratio of phase space available to the lens relative to that available to the MCMC chain. See text. 